Meta

Journal des traducteurs

Translators' Journal

\title{
La résolution de problèmes en traduction : quelques pistes
}

\section{Anna Gil-Bardají}

Volume 55, numéro 2, juin 2010

URI : https://id.erudit.org/iderudit/044240ar

DOI : https://doi.org/10.7202/044240ar

Aller au sommaire du numéro

\section{Éditeur(s)}

Les Presses de l'Université de Montréal

ISSN

0026-0452 (imprimé)

1492-1421 (numérique)

Découvrir la revue

Citer cet article

Gil-Bardají, A. (2010). La résolution de problèmes en traduction : quelques pistes. Meta, 55(2), 275-286. https://doi.org/10.7202/044240ar

\section{Résumé de l'article}

En traduction, comme dans d'autres disciplines, la notion de problème a suscite un intérêt croissant au cours des dernières années. Néanmoins, l'ensemble des travaux consacrés à l'analyse du problème de traduction est loin de constituer un axe de recherche homogène, ce qui est encore aggravé par le manque d'études synthétisant et contrastant les différents apports sur la question. Pour pallier cette lacune, le présent article analyse et met en lumière la notion de problème telle qu'elle a été développée par quelques spécialistes importants du processus de traduction. La première partie passe en revue la notion de problème dans d'autres disciplines. La seconde recueille et analyse les principales études relatives au problème de traduction et aux différentes approches. Elle établit également une relation entre les processus de résolution de problèmes en traduction et les stratégies de traduction. Notre contribution vise à une meilleure compréhension des processus de résolution de problèmes en traduction, et à présenter une rétrospective de travaux parmi les plus significatifs dans ce domaine.
Ce document est protégé par la loi sur le droit d'auteur. L’utilisation des services d’Érudit (y compris la reproduction) est assujettie à sa politique d'utilisation que vous pouvez consulter en ligne.

https://apropos.erudit.org/fr/usagers/politique-dutilisation/ 


\title{
La résolution de problèmes en traduction: quelques pistes
}

\author{
ANNA GIL-BARDAJí \\ Universitat Autònoma de Barcelona, Barcelone, Espagne \\ anna.gil.bardaji@uab.es
}

\begin{abstract}
RÉSUMÉ
En traduction, comme dans d'autres disciplines, la notion de problème a suscité un intérêt croissant au cours des dernières années. Néanmoins, l'ensemble des travaux consacrés à l'analyse du problème de traduction est loin de constituer un axe de recherche homogène, ce qui est encore aggravé par le manque d'études synthétisant et contrastant les différents apports sur la question. Pour pallier cette lacune, le présent article analyse et met en lumière la notion de problème telle qu'elle a été développée par quelques spécialistes importants du processus de traduction. La première partie passe en revue la notion de problème dans d'autres disciplines. La seconde recueille et analyse les principales études relatives au problème de traduction et aux différentes approches. Elle établit également une relation entre les processus de résolution de problèmes en traduction et les stratégies de traduction. Notre contribution vise à une meilleure compréhension des processus de résolution de problèmes en traduction, et à présenter une rétrospective de travaux parmi les plus significatifs dans ce domaine.
\end{abstract}

\begin{abstract}
In translation, as in other disciplines, the notion of problem has generated growing interest over recent years. However, studies centred on the translation problem taken as a whole, still do not represent a homogeneous research trend, and this is further aggravated by the lack of studies which compare and contrast the different contributions on this matter. In response to this need, this article analyses and develops the notion of problem as it has been described by some leading scholars reflecting on the translation process. In the first part, the notion of problem as seen from other disciplines is reviewed. In the second part, the main studies on the translation problem and different approaches are compared and analysed, and relate problem-solving processes in translation with translation strategies. The aim of this article is to further our understanding of the problem-solving processes in operation during translation, and to present a retrospective of some of the most significant works in this field.
\end{abstract}

\section{MOTS-CLÉS/KEYWORDS}

processus cognitifs, stratégie de résolution de problèmes, didactique de la traduction, théorie de la traduction

cognitives processes, problem-solving strategy, didactics of translation, translation theory

\section{Introduction}

La notion de problème a occupé, et continue d'occuper, une place significative en psychologie cognitive, ainsi que dans d'autres disciplines, comme les mathématiques ou la pédagogie. Cependant, l'intérêt pour comprendre la nature des processus en jeu dans la résolution de problèmes et la manière dont ceux-ci sont structurés dans notre pensée a ouvert récemment les portes à un ensemble encore plus vaste d'études 
concernant la connaissance humaine. La traduction en est un exemple significatif. En dépit de la difficulté liée à la définition du problème en traduction, et par conséquent à l'élaboration d'un cadre théorique adéquat pour sa résolution, la notion de problème a pris de plus en plus d'importance dans le domaine de la traduction, en raison, surtout, de la tendance croissante à considérer le problème comme le déclencheur de toute une série de processus mentaux. Le caractère stratégique de ces processus, leur rapport avec l'acquisition de la compétence traductrice et la didactique de la traduction, font de ce sujet une des clés dans la recherche actuelle en traduction.

\section{La notion de problème: une généalogie scientifique}

\subsection{Les origines scientifiques du problème}

Selon les psychologues de la Gestalt (Robine 2004; Ginger 1987/2003; Yontef 1993), le processus de résolution de problèmes consiste à essayer de mettre en relation un aspect d'une situation difficile avec un autre, ce qui a pour résultat une compréhension structurale, c'est-à-dire la capacité de saisir comment toutes les parties d'un problème s'emboîtent afin de satisfaire les exigences de l'objectif. Cela implique une réorganisation des éléments de la situation problématique de manière à résoudre le problème. Cette réorganisation suppose un processus complexe qui va de l'identification du problème à sa résolution et son évaluation.

Dans le domaine des mathématiques, l'une des principales approches de la résolution de problèmes fut celle du mathématicien Polya (1965). Selon lui, «la résolution de problèmes est basée sur des processus cognitifs qui ont pour résultat de trouver une sortie à une difficulté, une voie autour d'un obstacle, de manière à atteindre un objectif qui n'était pas immédiatement atteignable» (Polya 1965: 23). De Vega, quant à lui, entend, par résolution de problèmes, «les tâches qui exigent des processus de raisonnement relativement complexes, et non pas une simple activité associative et routinière» (De Vega 1984: 125, traduction de l'auteure).

Quant aux études concernant la résolution de problèmes appliquée à l'éducation, Pozo, del Puy Perez et al. (1994) partent de la distinction entre problème et exercice. Selon eux, un problème se différencie d'un exercice par le fait que, dans ce dernier cas, nous disposons de mécanismes que nous utilisons pour nous conduire immédiatement à la solution. Par contre, un problème est une situation nouvelle ou différente de celles qui ont été apprises, qui requiert l'utilisation stratégique de techniques connues au préalable (Pozo, del Puy Perez et al. 1994: 17-18). En ce sens, si un problème est résolu à plusieurs reprises, il finit par se convertir en exercice. Ainsi donc, pour la résolution de problèmes entrent en jeu des stratégies, une prise de décision, etc., tandis que pour la solution d'exercices, sont exercées des habilités acquises au préalable. Cette distinction entre problème et exercice avait déjà été faite par Polya, séparant les stratégies ou heuristiques des autres procédés de résolution de problèmes comme les règles ou les algorithmes. Alors que ce dernier type de procédés constitue des connaissances acquises qui permettent de transformer l'information d'une manière fixe, efficace et précise, les stratégies ou heuristiques guident la résolution de problèmes de manière beaucoup plus vague et globale (Polya 1965: 32).

D’autre part, le psychologue Sternberg a décrit plus récemment le processus de résolution de problèmes comme un processus cyclique qui irait de l'identification du 
problème à l'évaluation du processus complet qui doit être mis en œuvre pour sa résolution (Sternberg 1990). En ce qui concerne l'application de ressources, Sternberg fait référence aux procédés mentaux ou opérationnels mis en œuvre afin de résoudre un problème déterminé. Ces procédés peuvent être, selon l'auteur, de deux types: algorithmiques, c'est-à-dire basés sur une séquence d'opérations qui nous conduit à la solution, ou heuristiques, c'est-à-dire basés sur une recherche sélective, plus intuitifs et discriminatoires.

\subsection{Stratégies globales et stratégies spécifiques pour la résolution de problèmes}

Pozo, del Puy Perez et al. (1994) distinguent deux tendances générales en ce qui concerne le cheminement vers la résolution de problèmes et son application à l'enseignement. Nous considérons qu'une telle distinction, outre sa valeur synthétique et explicative, constitue un cadre très approprié pour étudier les processus de résolution de problèmes en traduction, et les appliquer en classe.

La première tendance, selon les auteurs, est celle qui a été adoptée assez longtemps par les études de psychologie appliquée à l'éducation. Selon cette tendance, la résolution de problèmes est basée sur l'acquisition de stratégies globales qui, dès qu'elles sont acquises, peuvent être appliquées, à quelques restrictions près, à tout type de problèmes, indépendamment de leur nature. De ce point de vue, enseigner la résolution de problèmes consiste à fournir aux élèves ces stratégies générales afin qu'ils puissent les appliquer chaque fois qu'ils se trouvent devant une situation nouvelle et problématique (Pozo, del Puy Perez et al. 1994: 20). Cette première tendance a donné lieu à différents types de classifications de problèmes, la plus importante étant celle qui distinguait les problèmes bien définis et les problèmes mal définis. Les problèmes bien définis sont ceux dont l'objectif est bien connu dès le début, alors que les problèmes mal définis sont ceux pour lesquels la définition des objectifs est déjà en soi un problème. De Vega (1984), se basant sur cette même classification, les nomme problèmes bien structurés ou mal structurés.

La seconde tendance, plus récente, considère que la résolution de problèmes et son enseignement ne peuvent être abordés que dans le contexte des domaines ou des contenus spécifiques correspondant à ces problèmes: dans ce cas, la résolution de problèmes est liée à la matière ou discipline à laquelle ils appartiennent (par exemple, comment résoudre des problèmes mathématiques, médicaux, linguistiques, etc.). Les études habituellement réalisées en ce sens consistent à comparer la manière dont les experts et les novices résolvent un problème relevant d'un domaine spécifique. Le fait de considérer la résolution de problèmes comme un processus spécifique a fait dernièrement l'objet d'un large consensus dans des domaines nombreux et variés de connaissance, des plus traditionnels (mathématiques, physique, logique) aux plus récents (médecine moderne, sciences infirmières, enseignement des langues étrangères, traduction, etc.). Ainsi,

[1]a première et la plus essentielle des hypothèses prises en compte dans les études sur la résolution de problèmes par des experts et des novices, c'est que les habiletés et les stratégies de résolution de problèmes sont spécifiques à un domaine déterminé et, par conséquent, difficilement transférables d'une spécialité à l'autre (Pozo, del Puy Perez et al. 1994: 35-36, traduction de l'auteure). 
L'efficacité dont fait preuve un expert dans la résolution de problèmes résulte de ses connaissances spécifiques. En fait, il semble que la compétence implique une utilisation optimale des ressources cognitives disponibles dans le domaine de spécialité en cause. En outre, Pozo, del Puy Perez et al. (1994) constatent que les habiletés nécessaires à la résolution d'un problème, et en général la compétence, résultent de la pratique. Par conséquent, non seulement il faut s'entraîner à résoudre des problèmes, mais une pratique intensive est nécessaire à l'obtention de résultats.

Néanmoins, les études se fondant sur un modèle opposant les comportements de l'expert et du novice ont été fréquemment critiquées, car elles insistent trop sur l'aspect quantitatif de la pratique professionnelle. On affirme, par exemple, que pour résoudre des problèmes dans un domaine déterminé, il est indispensable d'avoir essayé, si possible avec succès, de solutionner de nombreux problèmes relevant de ce domaine (Pozo, del Puy Perez et al. 1994). Néanmoins, il faut bien préciser que tous les types de pratiques ne sont pas également efficaces. Ce qui caractérise habituellement l'expérience d'un bon expert, ce n'est pas tant la quantité de problèmes résolus (nécessaire mais non suffisante) que le fait que la pratique est guidée par des principes conceptuels qui lui donnent un sens.

Nous verrons plus loin comment les études sur la résolution de problèmes appliquées à la didactique de la traduction ont évolué parallèlement à cette seconde tendance. Il faut par ailleurs rappeler que, jusqu'à la création de centres d'enseignement spécialisé, la pratique professionnelle a été la seule voie de formation des traducteurs: des milliers d'heures de pratique ou d'exercice, concentrées sur au moins dix ans d'expérience intensive, étaient considérées comme nécessaires pour accéder au statut d'expert.

\subsection{La résolution stratégique de problèmes}

Un point clé dans les études sur les processus de résolution de problèmes est le caractère stratégique que doivent avoir lesdits processus pour être effectifs. Selon Pozo, del Puy Perez et al. :

La résolution de problèmes requiert que l'entraînement technique soit complété par une connaissance stratégique qui permette l'utilisation délibérée de ces techniques dans le contexte de tâches ou de situations ouvertes, qui admettent des solutions différentes (Pozo, del Puy Perez et al. 1994: 37, traduction de l'auteure).

En ce sens, de nombreuses études concernant les processus de résolution de problèmes ont montré non seulement que les experts sont plus rapides et commettent moins d'erreurs, mais, en outre, qu'ils adoptent des stratégies différentes de celles auxquelles les novices ont recours. Il ressort des recherches portant sur la résolution de problèmes bien définis que les experts investissent habituellement moins de temps dans la résolution d'un problème de leur domaine de connaissance parce qu'ils reconnaissent plus facilement le problème comme correspondant à une situation connue, de sorte qu'ils établissent, plus ou moins automatiquement, le plan d'action adéquat qu'ils exécutent rapidement et efficacement.

En ce qui concerne l'acquisition d'habiletés spécifiques dans une matière déterminée, Anderson (1983) affirme que la résolution experte d'un problème implique la conversion de connaissances verbales ou déclaratives, consistant en instructions ou 
en règles, en une séquence de procédés de traduction appliqués de manière rapide et automatisée, autrement dit, des connaissances opératives.

Cette question est essentielle à notre réflexion, puisque ce que l'on souhaite pour l'enseignement de la traduction, grâce à l'étude de ces processus stratégiques, c'est précisément passer de connaissances éminemment opératives à des connaissances déclaratives. Néanmoins, définir précisément ces connaissances opératives n’est pas exempt d'obstacles, étant donné la difficulté à accéder aux processus mentaux.

Il est important de signaler, à ce sujet, que la résolution experte de problèmes est en grande partie fondée sur l'application automatique de techniques, plus que sur l'usage délibéré et intentionnel de stratégies. Cependant, cette automatisation, produit de la pratique, permet de libérer des ressources cognitives qui font que la conduite experte est plus efficace quand elle est confrontée à de vrais problèmes, c'està-dire à des situations qui ne peuvent être facilement réduites à des catégories connues au préalable. L'avantage qu'ont les experts à l'égard de ces stratégies non automatiques semble résider dans le fait qu'ils exercent un plus grand contrôle sur le processus de résolution de problèmes.

\section{La notion de problème en traduction}

Les problèmes en traduction, comme bien souvent pour l'ensemble des sciences humaines et sociales, sont des problèmes difficiles à définir. Par conséquent, l'élaboration d'un cadre théorique pertinent est complexe. Wilss affirme: «TS (Translation Studies) has had, and still does have, great trouble in defining a suitable and reliable conceptual framework for problem-solving» (Wilss 1996: 43).

Il est toutefois certain que la notion de problème ne cesse de gagner de l'importance en traduction, spécialement à cause de la tendance croissante à considérer le problème comme le moteur déclenchant toute une série de processus mentaux. C'est donc la pierre de touche de multiples études, en particulier celles qui concernent la didactique de la traduction. Or, malgré son importance, la notion de problème n'a pas été traitée de manière exhaustive. Seuls quelques auteurs se sont occupés de la question. Hurtado Albir (2001) signale à ce sujet que «nous ne disposons pas de définition du problème de traduction qui obtienne un certain consensus, ni d'une classification des problèmes de traduction qui ait été validée empiriquement» (Hurtado Albir 2001 : 280, traduction de l'auteure). Dans la même ligne, Presas (2000) ajoute:

Sans vouloir nier la valeur exemplaire de ces approches pratiques, nous devons signaler que toutes partent implicitement de la notion générale de problème, c'est-à-dire qu'elles n'accordent pas d'attention à la définition du problème de traduction (Presas 1996: 61, traduction de l'auteure).

Malgré tout, les tentatives pour définir le problème de traduction et en déterminer les catégories continuent d'intéresser la pédagogie de la traduction, car la connaissance préalable des problèmes les plus fréquents, ainsi que les différentes manières de les résoudre, constituent des instruments essentiels pour la prévention des erreurs et l'accroissement des compétences. 


\subsection{Approches théoriques du problème en traduction}

En traductologie, on observe deux types d'approches de l'étude du problème. Les unes associent la notion de problème à un certain type de connaissances (par exemple, connaissance de la langue, des propriétés du texte, de la relation entre le texte de départ et le texte d'arrivée, etc.). Elles partent de l'hypothèse que le texte est composé de segments et qu'il est possible d'isoler ceux qui sont problématiques de ceux qui ne le sont pas. Les autres considèrent que le problème est lié à la capacité du traducteur, autrement dit, à des facteurs plutôt cognitifs ou liés aux processus cognitifs.

Pour les premières, Presas (2000), à partir de la linguistique du texte, propose une classification qui distingue les problèmes intratextuels (cohérence et cohésion), les problèmes extratextuels (informativité, situationnalité et acceptabilité) et les problèmes intertextuels (conventions de genre). Pour les secondes, Krings (1986) envisage une double distinction: d'une part, les problèmes de réception et les problèmes de formulation (ou la combinaison des deux) et d'autre part, les problèmes de compétence en L2 et les problèmes de compétence de traduction.

Parallèlement à ces deux tendances, les études concernant les problèmes de traduction ont vu s'ouvrir récemment une troisième voie de recherche: la relation entre créativité et résolution de problèmes. Mackenzie (1998) signale que la recherche sur la créativité et la résolution créative de problèmes distingue habituellement les problèmes fermés des problèmes ouverts. Le fait que beaucoup de problèmes auxquels sont confrontés les traducteurs soient ouverts, c'est-à-dire qu'ils ne possèdent pas de solution prédéterminée, implique par définition, selon Mackenzie, l’application de stratégies créatives.

\subsection{Relations entre les stratégies de traduction et le problème de traduction}

C'est sans doute dans le cadre des études concernant les stratégies de traduction que la notion de problème a cependant exercé une plus grande influence. La relation de cause à effet établie entre problème et stratégie a modifié substantiellement la manière de concevoir les connaissances opératives du traducteur. Étant donné la relative modernité de cette nouvelle approche théorique (les études sur le problème de traduction ne remontent pas au-delà des trois dernières décennies), celle-ci constituera un point d'intersection conceptuel important entre ce que les premières recherches ont appelé procédés ou techniques et ce qui plus tard a été dénommé stratégies.

L'un des premiers à s'être intéressé au problème de traduction à partir de la linguistique textuelle fut de Beaugrande (1978). Très tôt, il a eu l'intuition de l'extrême importance du rôle joué par la notion de problème, sur les plans conceptuel et méthodologique, pour l'étude des stratégies et des techniques de traduction. Dans sa description des trois types de stratégies guidant le processus de traduction, de Beaugrande explique que celles-ci répondent aux contraintes imposées par le texte. Toutefois, l'auteur s'empresse de préciser que, logiquement, les stratégies activées par le contexte ne résoudront pas tous les problèmes. Selon son opinion, il est inadéquat d'exiger qu'une théorie de la traduction apporte des solutions à tous les problèmes de traduction. Par contre, elle devrait pouvoir offrir les principes et les stratégies nécessaires pour les aborder (de Beaugrande 1978: 14). 
Cependant, le plus important est sans doute la classification des facteurs conduisant à différents types de non-équivalences que de Beaugrande établit à l'issue de son étude:

A theoretical model is needed for co-ordinating these 3 levels (language system, poetic use of language in texts and translator as reader/writer). In particular, equivalence can be obtained only by identifying and overcoming a complex spectrum of potential problems on these three levels (de Beaugrande 1978: 13, italiques de l'auteure).

Trois groupes sont ainsi définis: (a) les facteurs relevant des systèmes linguistiques; (b) les facteurs relevant de l'usage poétique du langage dans un texte concret; (c) les facteurs relevant du traducteur en tant que lecteur/écrivain.

Dans le groupe (a), de Beaugrande mentionne le caractère arbitraire de la relation entre les traits linguistiques et l'expression, les incompatibilités entre les systèmes grammaticaux, la non-correspondance des représentations informationnelles dans les textes et la non-correspondance entre les environnements sociolinguistiques des sujets parlants. Dans le groupe (b) sont inclus des problèmes tels que la polyvalence et la multifonctionnalité, l'expansion d'un usage grammatical et lexical non ordinaire et le non-respect des attentes du lecteur. Dans le groupe (c), qui concerne le traducteur, apparaissent la prédominance de l'information basée sur le lecteur (reader-based information) par rapport à l'information textuelle (text-based information), la compétence inadéquate du traducteur.

Quelques années plus tard, bien que sans utiliser le terme problème, Wotjak (1981) affirmera que les techniques de traduction sont motivées, c'est-à-dire qu'elles ne sont mobilisées que quand quelque chose requiert leur action. Cette affirmation suppose un pas essentiel pour l'évolution des théories de Vinay et Darbelnet (1958) et les premiers théoriciens du processus de traduction. Selon eux, les procédés techniques étaient tous «ces mécanismes qui entrent en jeu lors du passage d'une langue $A$ à une langue $B$ », indépendamment de leur motivation éventuelle. Wotjak envisage trois types principaux de causes qui motivent l'usage des techniques de traduction: (a) linguistiques; (b) métalinguistiques; et (c) extralinguistiques.

Selon l'auteur, le groupe (a) embrasse des «cas problématiques de traduction» comme la non-existence de certains phénomènes grammaticaux et d'unités lexicales, c'est-à-dire des "phénomènes d'équivalence zéro, réels ou apparents, dans le plan correspondant à la langue» (Wotjak 1981 : 204), ou encore la non-coïncidence quant à la fréquence d'usage. En ce sens, Wotjak (1981: 205; italiques et traduction de l'auteure) élabore une liste approximative et indicative de quelques «obstacles concrets auxquels est confronté le traducteur»:

1) absence de catégories correspondantes dans le domaine de la syntaxe en L2;

2) existence de plusieurs catégories en L2 pour une seule catégorie de la L1;

3) différences entre les possibilités de combinaison sémantico-syntaxique;

4) différences entre les signifiés sémantiques ou pragmatico-stylistiques;

5) différence de phraséologie entre les deux langues;

6) différentes propriétés macrostructurales des textes en tant que représentants de types de textes.

Quant aux causes métalinguistiques du groupe (b), Wotjak les définit comme étant des «situations problématiques relativement peu fréquentes dans l'activité de traduction, mais qui, dans des cas concrets, sont extrêmement difficiles à résoudre» 
(Wotjak 1981 : 206, italiques et traduction de l'auteure). Ce sont notamment les jeux de mots ou les blagues, l'usage d'un déterminé sociolecte ou dialecte, la rime et la métrique, l'insertion d'autres langues étrangères dans le texte, les phrases toutes faites, etc.

Le groupe (c), comprenant des causes extralinguistiques, est basé sur les «différences de fond socioculturel»(Wotjak 1981: 207, traduction de l'auteure), comme celles que nous trouvons dans les phénomènes sociaux, administratifs, géographiques, etc., ainsi que les allusions, le type d'humour, caractéristiques du milieu de la L1.

Partant d'une approche communicative, Faerch et Kasper (1983) affirment que ce qu'ils dénomment stratégies de communication sont en réalité des manières de résoudre des problèmes de communication. Ces stratégies de communication, selon les auteurs, sont essentiellement de deux types: les stratégies d'évitement (changer ou réduire le message, d'une manière ou d'une autre, afin d'éviter le problème) et les stratégies d'accomplissement (essayer de conserver le message mais en changeant la forme, par exemple à l'aide de paraphrases, de rapprochements, de restructurations, du mime, etc.).

Pour ces auteurs, mettre en relation les stratégies et la résolution de problèmes présuppose la distinction entre deux types d'objectifs: 1) ceux que l'individu n'a aucune difficulté à atteindre; 2) ceux qui deviennent des problèmes. En ce sens, ils affirment que ne peuvent être considérés comme stratégies que les plans d'action élaborés pour ce dernier type d'objectifs:

[...] the goal of a strategy (the "strategic goal") is the problem, and the product of the execution phase controlled by the strategy is a solution to the problem" (Faerch et Kasper 1983: 33; italiques de l'auteure).

Au cours des années 1990, à partir de sa définition quasi historique, de «stratégie de traduction» ("a translation strategy is a potentially conscious procedure for the solution of a problem which an individual is faced with when translating a text segment from one language to another ", Lörscher 1991: 76), Lörscher a jeté les bases du concept de stratégie, depuis lors, indissociable de la notion de problème. L'intérêt de l'étude de Lörscher réside dans sa distinction, au sein du processus de traduction, entre "les phases stratégiques» (strategic phases) et les "phases non stratégiques» (non-strategic phases), les premières consistant à résoudre des problèmes, et les secondes, à accomplir une tâche:

[...] the translation process contains both strategic phases, which are directed towards solving translational problems, and non-strategic phases, which aim to accomplishing tasks (Lörscher 1991: 119, italiques de l'auteure).

Cette distinction part de l'analyse des résultats obtenus à partir d'une étude empirique menée chez une cinquantaine de sujets à l'aide de protocoles de verbalisations (PV; Think-Aloud Protocols). Les PV ont permis de mettre en évidence que le processus de traduction était parfois interrompu par des pauses plus ou moins longues. Avec l'idée de classifier les causes de ces interruptions, Lörscher a considéré empiriquement qu'il y a problème lorsque ces pauses dépassent deux secondes. Cette représentation empirique du problème est essentielle dans la mesure où elle permet à l'auteur de délimiter le terrain d'étude relatif à la question de la stratégie, puisque celle-ci s'instaure dès la détection du problème et s'achève à la résolution ou à la nonrésolution de ce dernier. Soulignons que Lörscher estime qu’un problème est résolu 
lorsque le sujet traduisant le considère ainsi (même si la solution à laquelle il parvient est, objectivement, erronée).

L'opposition entre les phases stratégiques et les phases non stratégiques est parallèle à celle qui existe entre les stratégies de traduction (translation strategies) et les versions de traduction (translation versions). Selon Lörscher, alors que les stratégies de traduction ne peuvent être mises en œuvre, par définition, que pendant les phases stratégiques du processus de traduction, les versions, quant à elles, peuvent être observées au cours des deux phases, ou encore s'étendre d'une phase stratégique vers une phase non stratégique ou vice-versa (Lörscher 1991: 119). Ces versions de traduction ont lieu, selon les données obtenues par Lörscher, dans des situations où, par exemple, le sujet n'arrive pas à résoudre un problème du premier coup et le laisse pour plus tard.

Comme Lörscher, Kiraly (1995) utilise les PV et se base sur les résultats obtenus pour distinguer les unités problématiques (problem units) des unités non problématiques (non problem units). Les premières requièrent, selon l'auteur, plus d'attention sur le plan cognitif et l'application de stratégies conscientes ou potentiellement conscientes. Les secondes présentent des solutions plus spontanées et intuitives, apparemment sans l'intervention de stratégies de résolution de problèmes.

D'autre part, Kiraly distingue également les processus soumis à un contrôle accru (c'est-à-dire plus conscients) des processus soumis à un contrôle moindre (c'est-à-dire plus intuitifs). Les premiers (situés dans ce que Kiraly, suivant Boekaerts [cité dans Kiraly 1995], appelle controlled processing center) sont des processus de type stratégique, c'est-à-dire qu'ils servent à résoudre de manière plutôt consciente les problèmes qui apparaissent dans la traduction. Les seconds, par contre (Kiraly les situe dans ce qu'il appelle intuitive workspace, qui remplace ce que Boekaerts (1997) nomme subconscious workspace), sont des processus plutôt automatisés et intuitifs. Lorsque les traitements automatisés ne sont plus capables de produire des éléments de traduction, les problèmes émergent dans l'intuitive workspace et passent alors au controlled processing center, là où sont choisies les différentes stratégies de résolution. Mais, avertit Kiraly, les stratégies ne permettent pas de résoudre les problèmes de traduction. Ce ne sont que des plans d'action prévus pour les résoudre (Kiraly 1995: 149). De toute manière, la relation d'antériorité existant entre les processus non contrôlés et les problèmes de traduction n'est pas toujours évidente:

The data analysis showed that only some translation problems appeared to be focused upon for controlled processing. The verbalizations revealed that potentially conscious strategies were implemented only when subjects were unable to produce an acceptable translation solution for a source text element through spontaneous association or other unidentifiable uncontrolled processes. The absence of logical sequences of controlled processes leading to accepted solutions suggests that translation strategies are used to provide material for uncontrolled processes. It is significant that the final decision made in the processing chain for each translation unit was in almost every case an intuitive one (Kiraly 1995: 143).

Hönig (1991), quant à lui, propose ses propres conceptions: les micro-stratégies (microstrategies) et les macro-stratégies (macrostrategies), dont l'application quasiautomatique est liée à la compétence du traducteur:

The translator will become aware of his translation task(s) by collecting and collating data from his uncontrolled workspace, the prospective $t$ and the projected st. This leads 
to the development of a translation macro-strategy, which professional translators may apply almost automatically on the basis of their professional experience, or very deliberately, possibly with the aid of translation-relevant textual analysis (Hönig 1991: 80; italiques de l'auteure).

Quant aux microstratégies, Hönig les situe dans le controlled workspace:

The controlled workspace could be defined as the location where all those mentalcognitive processes take place which find their way into thinking aloud protocols. They are basically monitoring strategies and/or the application of micro-strategies (isolated rules) (Hönig 1991: 80; italiques de l'auteure).

Néanmoins, Hönig émet des réserves à propos de ce qui différencie réellement certains termes concernant les processus mentaux du traducteur, tels que intuitif/ cognitif (intuitive/cognitive), conscient/inconscient (conscious/unconscious) et contrôlé/ non contrôlé (controlled/uncontrolled) : ainsi, l'opposition intuitif/cognitif ne serait pas pertinente, comme il le montre dans le cadre d'une étude réalisée avec des semiprofessionnels, dont l'objectif était de déterminer comment ils parvenaient à la solution finale. Les résultats furent les suivants:

In the vast majority of cases this happened through an un co-ordinated sequence of intuitive and cognitive steps, so that the whole chain could be termed neither intuitive nor cognitive (Hönig 1991: 81).

Séguinot (1991), quant à elle, jette une nouvelle lumière sur les études concernant les stratégies et la résolution de problèmes, en mettant celles-ci en rapport avec la compétence de traduction. L'auteure part de la prémisse selon laquelle, en dehors de leur plus ou moins grande capacité à résoudre des problèmes, les experts et les novices traitent et récupèrent l'information de manière différente. Sa position concorde avec celle de Dreyfus et Dreyfus (1986: 16-51), lorsque ces derniers suggèrent que le modèle de prise de décisions (qui est habituellement appliqué à la traduction) ne représente qu'une proportion très petite du comportement réel de l'expert. L'auteure conclut en affirmant:

While competent behavior may involve conscious deliberation, the level above that, i.e. the level of experts, seems to involve a holistic recognition of similarities between novel situations and stored experience rather than problem-solving (Séguinot 1991: 81).

Par contre, Kussmaul (1995) considère que c'est par l'étude des erreurs que l'on peut arriver à déterminer quelles sont les techniques et les stratégies à utiliser afin de les éviter et, par conséquent, atteindre une plus grande compétence. En ce sens, Kussmaul mise sur l'élaboration d'une typologie de stratégies et de techniques qui pourraient résoudre certaines erreurs de traduction.

Andrew Chesterman (1997) affirme également que les stratégies ont pour origine un problème:

If the goal is the end-point of a strategy, what is the starting point? The simple answer is: a problem. A strategy offers a solution to a problem, and is thus problem-centered [...] The translation process too starts with problems (Chesterman 1997: 89).

Selon Chesterman, les études empiriques réalisées menées auprès de traducteurs, à l'aide de PV ou d'autres méthodes similaires, démontrent que ceux-ci ont tendance à agir par à-coups (jerks). En d'autres termes, alors que la traduction se déroule sans 
incidents à certains moments, un obstacle supposé arrête la marche naturelle du processus à d'autres. Chesterman considère que c'est essentiellement lorsqu'ils rencontrent de tels obstacles que les traducteurs ont recours à des stratégies.

Enfin, un axe de recherche récent, de même orientation, consiste à étudier le caractère créatif des stratégies en traduction et en interprétation (voir BeylardOzerof, Králová, et al. 1998). Mackenzie (1998), par exemple, considère que la résolution de problèmes est une activité essentiellement créatrice, même dans les situations où cela paraît le moins évident. Cette affirmation repose sur la théorie qui conçoit la traduction comme un processus de résolution de problèmes: l'habileté du traducteur réside dans sa capacité à appliquer des stratégies appropriées pour résoudre des problèmes déterminés. Dans ce cadre, Mackenzie signale deux types de problèmes traditionnels, ouverts ou fermés. De nombreux problèmes auxquels sont confrontés les traducteurs sont ouverts, c'est-à-dire qu'ils ne possèdent pas de solution prédéterminée, qu'ils ne peuvent être résolus consciemment dans des conditions contrôlées et que leurs solutions ne sont pas sujettes à une vérification absolue. Par conséquent, les résoudre requiert des stratégies créatives par nature. Wallis (1981 : 24; cité par Mackenzie [1998: 201]) énumère les différentes étapes de ce processus créatif:

1) préparation (recueil de l'information);

2) incubation (mise en œuvre d'un type de travail mental inconscient);

3) illumination (émergence de la solution);

4) vérification (vérification et élaboration de solutions).

\section{Conclusion}

L'objectif du présent article était d'analyser la notion de problème de traduction à l'aide d'études pionnières qui se sont penchées sur le processus de traduction, ainsi que que d'en faire la synthèse. Les résultats de cette analyse montrent, premièrement, que les recherches autour de la notion de problème entreprises au sein de plusieurs disciplines, notamment la psychologie cognitive et la pédagogie, trouvent dans la traductologie un champ fertile. Deuxièmement, ils permettent de constater l'importance de la notion de stratégie dans les processus de résolution de problèmes de traduction, ce qui autorise l'établissement d'un lien entre deux axes de recherche fondamentaux au sein des études de traduction: d'un côté, celle qui s'intéresse aux processus de résolution de problèmes, et de l'autre, celle qui s'intéresse à la didactique de la traduction.

\section{RÉFÉRENCES}

Anderson, John Robert (1983): The Architecture of Cognition. Cambridge: Harvard University Press.

Beylard-Ozerof, Ann, Králová, Jana et Moser-Mercer, Barbara, dir. (1998): Translation Strategies and Creativity. Amsterdam: John Benjamins.

BoekaerTs, Monique (1997): Self-regulated learning: a new concept embraced by researchers, policy makers, educators, teachers, and students. Learning and Instruction. 7(2):151-186.

Chesterman, Andrew (1997): Memes of Translation. Amsterdam: John Benjamins. De Beaugrande, Robert (1978): Factors in a Theory of Poetic Translation. Assen: Van Gorcum. De vega, Manuel (1984): Introducción a la psicología cognitiva. Madrid: Alianza Psicología. 
Dreyfus, Hubert et Dreyfus, Stuart (1986): Mind over Machine. Oxford: Basil Blackwell.

FAERCH, Claus et KASPER, Gabrielle (1983): Plans and strategies in foreign language communication. In: Claus FAERCH et Gabrielle KASPER, dir. Strategies in Interlanguage Communication. New York: Longman, 20-60.

Ginger, Serge et Anne (1987/2003): La Gestalt, une thérapie du contact. Paris: Hommes et groupes éditeur.

HöNIG, Hans (1991): Holmes' "mapping theory" and the landscape of mental translation processes. In: Kitty van Leuven-Zwart et Ton NaAijkens, dir. Translation Studies: The State of the Art. Amsterdam: Rodopi, 77-89.

Hurtado Albir, Amparo (2001): Traducción y traductología: introducción a la traductología. Madrid: Cátedra.

Kiraly, Donald (1995): Pathways to Translation, Pedagogy and Process. Kent: The Kent State University Press.

KRINGS, Hans (1986): Was in den Köpfen von Übersetzern vorgeht. Eine empirische Untersuchung der Struktur des Übersetzungsprozesses an fortgeschrittenen Französischlernern. Tübingen: Narr.

Kussmaul, Paul (1995): Training the Translator. Amsterdam: John Benjamins.

LöRSCHER, Wolfgang (1991): Translation Performance, Translation Process and Translation Strategies: A Psycholinguistic Investigation. Tübingen: Gunter Narr Verlag.

Mackenzie, Rosemary (1998): Creative problem-solving and translator training. In: Ann Beylard-Ozerof, Jana Králová et Barbara Moser-Mercer, dir. Translation Strategies and Creativity. Amsterdam: John Benjamins, 201-206.

Polya, George (1965): Poser et résoudre un problème. Paris: Dunod.

Pozo, Juan Ignacio, del Puy Perez, Maria, Dominguez, Jesús et al. (1994): La solución de problemas. Madrid: Santillana.

Presas, Marisa (2000): Bilingual competence and translation competence. In: Christina Schäffner and Beverly AdAB, dir. Developing Translation Competence. Amsterdam: John Benjamins, 19-31.

RoBINE, Jean-Marie (2004): S'apparaître à l'occasion d'un autre: études pour la psychothérapie. Bordeaux: L'Exprimerie.

SÉGuinot, Candace (1991): A study of student translation strategies. In: Sonja TirkkonenCondit, dir. Empirical Research in Translation and Intercultural Studies. Tübingen: Gunter Narr Verlag, 79-88.

Sternberg, Robert (1990): Metaphors of Mind: Conceptions of the Nature of Intelligence. New York: Cambridge University Press.

Vinay, Jean-Paul et Darbelnet, Jean (1958): Stylistique comparée du français et de l'anglais. Paris: Didier.

Wilss, Wolfram (1996): Knowledge and Skills in Translator Behavior. Amsterdam: John Benjamins.

Wotjak, Gerd (1981): Técnicas de translación. In: Mario Medina, Leandro Caballero et Fernando Martínez, dir. Aspetos fundamentales de la teoría de la traducción. La Habana: Ediciones Pueblo y Educación, 197-229.

Yontef, Gary (1993): Gestalt Therapy: An Introduction. In: Gary Yontef. Awareness, Dialogue, and Process. Gouldsboro: The Gestalt Journal Press. 